\title{
The QCD static potential in $2+1$ dimensions at weak coupling
}

\author{
Maximilian Stahlhofen ${ }^{\mathrm{a}, *}$ \\ ${ }^{a}$ Grup de Física Teòrica and IFAE, Universitat Autònoma de Barcelona, E-08193 Bellaterra, Barcelona, Spain.
}

\begin{abstract}
Using the effective theory pNRQCD we determine the potential energy of a color singlet quark-antiquark pair with (fixed) distance $r$ in three space-time dimensions at weak coupling $(\alpha r \ll 1)$. The precision of our result reaches $O\left(\alpha^{3} r^{2}\right)$, i.e. NNLO in the multipole expansion, and NNLL in a $\alpha / \Delta V$ expansion, where $\Delta V \sim \alpha \ln (\alpha r)$. We even include all logarithmic terms up to $\mathrm{N}^{4} \mathrm{LL}$ order and compare the outcome to existing lattice data.
\end{abstract}

Keywords:

static potential, $2+1$ dimensions, pNRQCD, ultrasoft corrections

\section{Introduction}

The potential energy of a static quark-antiquark pair in the color singlet at short distances $r$ is an essential ingredient in the theoretical description of heavy quarkonium. Its large-distance behavior, which is probed e.g. in lattice simulations, indicates confinement. The determination of the static potential in three space-time dimensions (3D) represents an important check of the methods used for the four-dimensional (4D) calculation [1-3]. The results can also be applied within thermal QCD. In this paper we determine the 3D static potential for $\alpha r \ll 1$ using the effective field theory "potential nonrelativstic QCD" (pNRQCD) [4, 5]. Unlike conventional perturbative QCD, this effective theory framework allows for so-called ultrasoft effects, which are crucial for consistent results beyond one loop [68]. We discuss the renormalization group structure of the 3D static potential and present recent results up to $O\left(\alpha^{3} r^{2}\right)$ and partly $\mathrm{N}^{4} \mathrm{LL}$ precision. We also compare these results to existing lattice data. The work presented here is based on Ref. [9].

\section{Theoretical preliminaries}

Because in $D$ dimensions the mass dimension of the coupling is $\left[g^{2}\right]=M^{4-D}, g^{2} r^{4-D}$ is a dimensionless (expansion) parameter and we have (at least) the following physical scales involved in the problem: $1 / r$ (soft),

\footnotetext{
*Speaker

Email address: stahlhofen@ifae.es (Maximilian Stahlhofen)
}

Preprint submitted to Nuc. Phys. (Proc. Suppl.)
$V \sim 1 / r \times g^{2} r^{4-D}$ (ultrasoft), $g^{\frac{2}{4-D}}$ (non-perturbative). In order for perturbation theory at the soft scale and the pNRQCD multipole expansion to make sense we demand $g^{2} r^{4-D} \ll 1$, i.e. weak coupling.

For $D=3$ at short distances we find $V \sim g^{2} \ln (r v)$, where $v$ is the pNRQCD renormalization scale. This implies that the ultrasoft expansion parameter $g^{2} / V \sim$ $1 / \ln (r v) \ll 1$, if we choose $v \sim V$. We conclude that we can use perturbation theory at the ultrasoft scale $V$. Therefore we formally distinguish between the scales $V$ and $g^{2}$. Logarithms from the ultrasoft perturbative computation will have the form $\ln (V / v)$ and are rendered small, if we set $v \sim V$. Thus it is legitimate to consider the ultrasoft regime as perturbative, i.e. the pNRQCD loop expansion makes sense (for sufficiently small $r$ ). In the following we will use the index " $B$ " to explicitly denote bare quantities. Parameters without this index are understood to be renormalized in the MS scheme. Throughout this paper we will use the notation $D=$ $3+2 \epsilon$. In position space the bare singlet potential can be schematically written as

$$
V_{s, B} \equiv-C_{F} g_{B}^{2} \sum_{n=0}^{\infty} \frac{g_{B}^{2 n} c_{n}(D) r^{-2(n+1)\left(\epsilon-\frac{1}{2}\right)}}{r} .
$$

The singlet static energy can be considered to be an observable for our purposes. It consists of the potential, which is a pNRQCD Wilson coefficient, and an ultrasoft contribution 1 , either bare or renormalized:

$$
E_{s}(r)=V_{s, B}+\delta E_{s, B}^{u s}=V_{s}+\delta E_{s}^{u s} .
$$

\footnotetext{
${ }^{1}$ If one has enough precision also non-perturbative effects at the scale $g^{2}$ should be included. We will address them in Sec. 3
}

November 12, 2018 


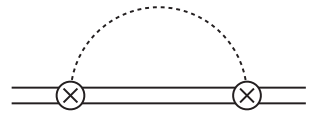

Figure 1: One-loop contribution to the octet propagator. The dotted line represents the $A^{0}$ component of the ultrasoft gluon field.

The soft contribution $V_{s, B}$ equals the purely perturbative bare static potential, which was computed in Ref. [10] up to two loops, i.e. $O\left(\alpha^{3} r^{2}\right)$. It is IR divergent at this order. Using pNRQCD in the static limit the ultrasoft contribution can be expressed in a compact form at NLO in the multipole expansion (but exact to any order in the ultrasoft loop expansion) through the chromoelectric correlator. It reads (in the Euclidean)

$$
\begin{aligned}
\delta E_{s, B}^{u s}= & V_{A}^{2} \frac{T_{F}}{(D-1) N_{c}} \mathbf{r}^{2} \int_{0}^{\infty} d t e^{-t \Delta V_{B}} \\
& \times\left\langle v a c\left|g_{B} \mathbf{E}_{E}^{a}(t) \phi_{\mathrm{adj}}^{a b}(t, 0) g_{B} \mathbf{E}_{E}^{b}(0)\right| v a c\right\rangle,
\end{aligned}
$$

where we have defined $\Delta V \equiv V_{o}-V_{s}$. The concrete result for the ultrasoft correction in $D$ dimensions up to two loops is given in Ref. [9]. It is known at one loop since Ref. [11] (see also [7, 12]) and was deduced at two loops from the results obtained in Refs. [13, 14].

The renormalized coupling constant $\alpha=\frac{g_{B}^{2} v^{2 \epsilon}}{4 \pi}$ has integer mass dimension and does not run in three dimensions as a consequence of the super-renormalizability of the theory.

The bare potentials $V_{B}$ in position space also have integer mass dimensions and following Ref [15] we define

$$
V_{B}=V+\delta V .
$$

$\delta V$ will generally depend on $\alpha$ and $V$. In the MS renormalization scheme it takes the form

$$
\delta V=\sum_{n=1}^{\infty} \frac{Z_{V}^{(n)}}{\epsilon^{n}},
$$

from which we can derive the RGE's for the different renormalized potentials $V$ in the usual way.

In pNRQCD at leading order in the multipole expansion the singlet field of the quark-antiquark system is free, i.e. it does not interact with gluons. It is therefore renormalization scale independent at $O\left(r^{0}\right)$. Similarly the singlet potential is not renormalized at this order:

$$
\delta V_{s}=O\left(r^{2}\right)
$$

For the octet field the situation is different. Even at leading order in the multipole expansion it has a residual interaction with ultrasoft gluons. The octet potential receives an ultraviolet (UV) divergent correction from the one-loop self-energy diagram shown in Fig. 1 .

$$
Z_{V_{o}}^{(1)}=\frac{C_{A}}{2} \alpha+O\left(r^{2}\right) .
$$

Higher loop diagrams cannot contribute at $O\left(r^{0}\right)$. This is because the potentials must appear perturbatively (with positive powers) in the Z's. Since $\alpha$ has positive mass dimension, the potentials would appear with negative powers in higher loop corrections to Eq. (7), which is not allowed by renormalizability. By the very same reason the octet field does not require renormalization at $O\left(r^{0}\right)$. With a similar argument and Ref. [14] we find $V_{A / B}=1+O\left(\alpha^{2}\right)$.

Solving the corresponding RGE's and matching to the soft tree-level result we find from Eqs. (6) and (7):

$$
\Delta V(v)=-\alpha C_{A} \ln \left(r v e^{\gamma_{E} / 2} \sqrt{\pi}\right)+O\left(r^{2}\right)+O(\epsilon) .
$$

We now focus on the renormalization of $V_{s}$ beyond $O\left(r^{0}\right)$. The singlet potential is IR safe up to soft oneloop order. At two soft loops in dimensional regularization IR poles up to $O\left(1 / \epsilon^{3}\right)$ appear [10]. The ultrasoft computation in pNRQCD, i.e. Eq. (3), yields the counterterms

$$
\begin{aligned}
& Z_{V_{s}}^{(1)}=r^{2} \Delta V^{2} \alpha Z_{V_{s}}^{(1,1)}+r^{2} \Delta V \alpha^{2} Z_{V_{s}}^{(1,2)}+r^{2} \alpha^{3} Z_{V_{s}}^{(1,3)}, \\
& Z_{V_{s}}^{(2)}=r^{2} \Delta V \alpha^{2} Z_{V_{s}}^{(2,1)}+r^{2} \alpha^{3} Z_{V_{s}}^{(2,2)}, \\
& Z_{V_{s}}^{(3)}=r^{2} \alpha^{3} Z_{V_{s}}^{(3,1)} \\
& Z_{V_{s}}^{(n)}=0 \quad \forall \quad n>3,
\end{aligned}
$$

where the explicit expressions for the $Z_{V_{s}}^{(x, y)}$ are given in Ref. [9]. These are the complete $O\left(r^{2}\right)$ results. Eq. (12) reflects the super-renormalizability of the theory. Eqs. (9,11) are obtained as follows: $Z_{V_{s}}^{(1,1)}$ comes from the one-loop $1 / \epsilon$ divergence in Eq. (3) and fixes also $Z_{V_{s}}^{(2,1)}$ and $Z_{V_{s}}^{(3,1)}$ by RG consistency. $Z_{V_{s}}^{(1,2)}$ follows from the remaining $1 / \epsilon$ divergence in the ultrasoft two-loop computation for $\delta V_{s}$, once all subdivergences have been subtracted. From this result we derive $Z_{V_{s}}^{(2,2)}$ again by RG arguments. Because the respective divergent parts of the bare quantities in Eq. (2) have to cancel, we can now also determine the missing term $Z_{V_{s}}^{(1,3)}$ without actually performing the corresponding ultrasoft three-loop calculation [9]. This is possible since $Z_{V_{s}}$ must not contain terms with negative powers of $\Delta V \sim \alpha \ln (r v)$, which cannot be absorbed by the potential, cf. Eq. (1). The fact that the resulting $Z_{V_{s}}^{(1,3)}$ is indeed independent of $\Delta V$ is a non-trivial crosscheck of both, the soft and the ultrasoft calculations. Thus we have found the complete RG structure of $V_{s}$ at $O\left(r^{2}\right)$. 


\section{Results}

From the counterterms determined in the previous subsection, we can derive the complete running of the singlet static potential at $O\left(r^{2}\right)$. By solving the RG equations we obtain

$$
V_{s}(v)=V_{s}\left(r ; v=\frac{1}{r}\right)+V_{s}^{\mathrm{RG}}(r ; v),
$$

where $V_{s}^{\mathrm{RG}}(r ; v)$ is the running and $V_{s}\left(r ; v=\frac{1}{r}\right)$ is the initial matching condition, which we have determined using the bare soft data of Ref. [10]. The full MS results are given in Ref. [9]. Note that Eq. (13) is the complete RG improved static potential (i.e. the soft contribution to the static energy) up to $O\left(r^{2}\right)$. Adding the finite parts of $\delta E^{u s}$ at one and two loops after minimal subtraction to Eq. (13) and setting $v=\Delta V$ to resum potentially large ultrasoft logarithms we obtain

$$
\begin{aligned}
E_{S}(r) & =C_{F} \alpha \ln \left(r^{2} v_{s}^{2} \pi e^{\gamma_{E}}\right)+\frac{\pi}{4} C_{F}\left(7 C_{A}-4 n_{f} T_{F}\right) \alpha^{2} r \\
& +C_{F} \alpha^{3} r^{2}\left\{\frac{1}{6} C_{A}^{2} \ln ^{3}(r \Delta V)\right. \\
& +\frac{1}{4} C_{A}^{2}\left(2 \gamma_{E}-1-2 \ln 2\right) \ln ^{2}(r \Delta V) \\
& +\left[n_{f} T_{F}\left(C_{A}\left(2+\frac{19 \pi^{2}}{48}\right)+C_{F}\left(5-\frac{\pi^{2}}{2}\right)\right)-\left(n_{f} T_{F}\right)^{2} \frac{\pi^{2}}{8}\right. \\
& +C_{A}^{2}\left(\frac{13 \pi^{2}}{384}+\frac{1}{2} \gamma_{E}^{2}+\frac{1}{2} \ln ^{2} 2-\frac{1}{4} \gamma_{E}(1+4 \ln 2)\right. \\
& \left.\left.\left.-\frac{1}{4}(11+\ln \pi)\right)\right] \ln (r \Delta V)\right\}+O\left(\alpha^{3} r^{2} \ln ^{0}\right) .
\end{aligned}
$$

This is the full result for the static energy up to $O\left(\alpha^{2} r^{2}\right)$ and ultrasoft NNLL order expressed as a double expansion in $\alpha r$ (multipole) and $1 / \ln (r \Delta V)$ (ultrasoft).

The omitted $O\left(\alpha^{3} r^{2}\right)$ terms do not contain logarithms of $r \Delta V$. Eq. (14) is invariant under a change of $v$ up to $O\left(\frac{\alpha^{4} r^{2}}{\Delta V}\right)$. The dependence of $E_{s}(r)$ at tree level on the factorization scale $v_{s}$ is related to the $3 \mathrm{D}$ relic of the 4D pole mass renormalon and would cancel, if we add twice the heavy quark mass to Eq. (14). In this work it will however be of no importance. Finally we would like to note that the condition $v \equiv \Delta V(v)$, produces a $v$ independent scale that is nonperturbative in $\alpha$ and resums a certain class of logarithms, see Ref. [9].

Since for $v \equiv \Delta V(v)$ the ultrasoft logarithms vanish, we only have to add the (RG-scheme dependent) constant term

$$
\left.V_{s}\left(r ; v=\frac{1}{r}\right)\right|_{O\left(\alpha^{3}\right)}+C_{F} C_{A}^{2} r^{2} \alpha^{3} c_{2,0}
$$

to Eq. (14) to reach $\mathrm{N}^{3} \mathrm{LL}$ order. $c_{2,0}$ can be computed perturbatively, but requires a three-loop pNRQCD computation which has not been performed yet.
At even higher orders in the $\alpha / \Delta V$ expansion, nonperturbative effects start to contribute. In order to study these effects related to loop momenta $k \sim \alpha$, we integrate out the $\Delta V$ scale. This means integrating out the octet field and ultrasoft gluons. The degrees of freedom left are the singlet field and nonperturbative gluons with energy and momentum of order $\alpha$. The resulting Lagrangian, including the leading order nonperturbative effects at $O\left(r^{2}\right)$, reads

$$
\begin{aligned}
\mathcal{L}_{\mathrm{np}}= & \operatorname{Tr}\left\{\mathrm{S}^{\dagger}\left(i \partial_{0}-V_{s}(r)-\delta E_{s}^{u s}\right) \mathrm{S}\right\} \\
& -\frac{C_{n p}}{\Delta V} \operatorname{Tr}\left\{\mathrm{S}^{\dagger}(g \mathbf{E} \cdot \mathbf{r})^{2} \mathrm{~S}\right\}
\end{aligned}
$$

for the case without light fermions $\left(n_{f}=0\right)$ to which we restrict ourselves in the following 2 The coefficient of the nonperturbative operator in Eq. (16) is $C_{n p}=1$ at leading order in the $\frac{\alpha}{\Delta V}$ expansion. This result is obtained by matching to a pNRQCD tree-level diagram, where two gluons couple to the singlet field at $O\left(r^{2}\right)$.

The interaction with nonperturbative gluons produces a shift of the energy which is proportional to the $3 \mathrm{D}$ gluon condensate:

$$
\delta E_{s, B}^{n p}=\frac{r^{2}}{\Delta V_{B}} \frac{2 \pi}{N_{c}(D-1) D}\left\langle\alpha G_{\mu \nu}^{a} G^{\mu v, a}\right\rangle_{B} .
$$

The leading ultraviolet divergence of the gluon condensate has been calculated in perturbation theory at four loops [16]. The determination of the finite piece requires lattice simulations [17, 18] and a computation to change from the lattice to dimensional regularization [19]. Taking the result (in the Euclidean) from the last reference and renormalizing the bare expression in Eq. (17) in the MS scheme yields

$$
\begin{aligned}
\delta E_{s}^{n p}(v)= & -\frac{C_{A}^{3} C_{F} r^{2} \alpha^{4}}{\Delta V}\left[( \frac { 4 3 } { 6 } - \frac { 1 5 7 } { 3 8 4 } \pi ^ { 2 } ) \left(\ln \left[\frac{v}{C_{A} \alpha}\right]\right.\right. \\
& \left.\left.-\frac{1}{2}\left(\ln (16 \pi)+\gamma_{E}\right)-\frac{1}{8}\right)+2 B_{G}\right],
\end{aligned}
$$

where $B_{G}^{(S U(3))}=-0.2 \pm 0.4(\mathrm{MC}) \pm 0.4(\mathrm{NSPT})$. This result is of the same order as the ultrasoft four-loop contribution, i.e. $O\left(r^{2} \alpha^{4} / \Delta V\right)$. For $v \equiv \Delta V$ however, the $\ln \left[\frac{v}{C_{A} \alpha}\right]$ term in Eq. (18) is parametrically dominant compared to the latter and we will include it in the numerical analysis of our results in the next section.

\footnotetext{
${ }^{2}$ If we were to include light fermions there would also be operators $\propto \mathrm{S}^{\dagger} \bar{q} q \mathrm{~S}$. They could generate corrections to the static energy, due to the quark condensate, which are of the same parametric order as the purely gluonic ones.
} 


\section{Comparison to lattice data}

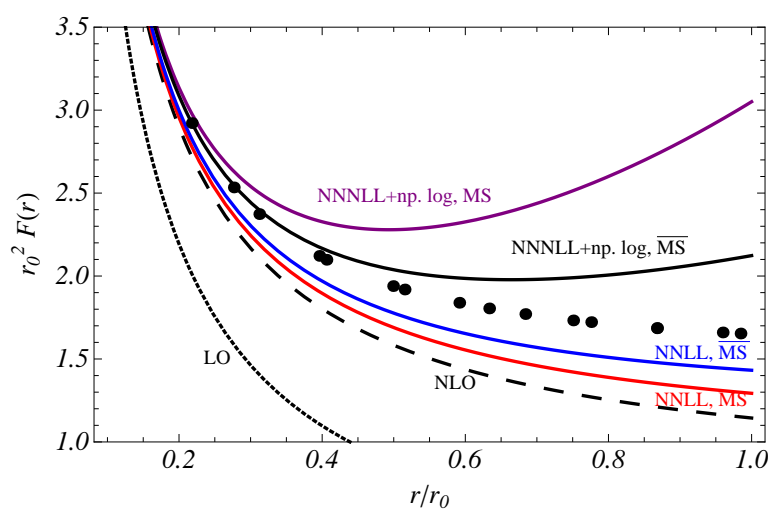

Figure 2: Plots of the analytic results for the force $F(r)=\frac{d E_{s}(r)}{d r}$ in " $r_{0}$ units" with $n_{f}=0$ in comparison to SU(2) lattice data (black dots): The dotted (LO) and dashed (LO+NLO) curves show the tree-level and one-loop results from Ref. [10]. The four other curves include in addition the new NNLO order $\left(\alpha^{3} r^{2}\right)$ contributions in the multipole expansion. The labels NNLL and $\mathrm{N}^{3} \mathrm{LL}$ refer to the precision in the ultrasoft $\alpha / \Delta V$ expansion. We have plotted our results in the MS as well as in the $\overline{\mathrm{MS}}$ scheme in order to make the scheme dependence visible and set $v=\Delta V$. Depending on the scheme we have used the values $c_{2,0}^{\mathrm{MS}}$ and $c_{2,0}^{\overline{\mathrm{MS}}}$ given in the text for the $\mathrm{N}^{3} \mathrm{LL}$ plots, which moreover incorporate the leading nonperturbative logarithm of Eq. 18.

Now we would like to see how well the short-distance 3D lattice data can be reproduced by our theoretical prediction for the static singlet energy and, on the other hand, extract numerical values for $c_{2,0}$ in Eq. (15) from fits to this data. In Fig. 2 we show our $O\left(\alpha^{3} r^{2}\right)$ MS results for the static force $F(r)=\frac{d E_{s}(r, r=\Delta V)}{d r}$ up to NNLL (from Eq. (14)) and up to $\mathrm{N}^{3} \mathrm{LL}$ including the leading nonperturbative logarithm (from Eq. (14) + Eq. (15) + first line of Eq. (18)) for $n_{f}=0$ and $N_{c}=2$ together with the data points from the $\mathrm{SU}(2)$ quenched lattice simulation of Ref. [20] 3 We use $\alpha=\frac{0.29}{r_{0}}$, where $r_{0}=0.5 \mathrm{fm}$ is the Sommer scale 4 To estimate the theoretical uncertainties we also transformed the MS results to the $\overline{\mathrm{MS}}$ scheme 5 and added the corresponding curves as well as the previously known curves at LO and NLO precision to the plot. From a fit of the NNLL $\overline{\mathrm{MS}}$ curve to the data point at the shortest distance, where we expect the best convergence of the perturbative series, we determined $c_{2,0}^{\overline{\mathrm{MS}}}=-0.04$. Transforming this to the MS scheme gives $c_{2,0}^{M S}=2.64$.

\footnotetext{
${ }^{3}$ There is also SU(3) lattice data available in Ref. [21], but it has less points at slightly larger distances.

${ }^{4}$ For the determination of $\alpha$ see Refs. [9, 20].

${ }^{5}$ Note, that this also implies $\Delta V_{\mathrm{MS}} \rightarrow \Delta V_{\overline{\mathrm{MS}}}$ in the logs of Eq. (14) etc. giving rise to a residual scheme dependence from higher orders.
}

If we compare the LO, NLO and the NNLO curves with ultrasoft NNLL precision, we find a convergent pattern, in particular in the short distance limit. Unlike the multipole expansion the $\alpha / \Delta V$ expansion does not converge well, even for the shortest distances that were probed on the lattice. Indeed, already at $r / r_{0} \simeq 0.22$ we have $\frac{C_{A} \alpha}{\Delta V_{\overline{\mathrm{MS}}}} \simeq 0.60\left(\lesssim \frac{C_{A} \alpha}{\Delta V_{\mathrm{MS}}}\right)$ for the ultrasoft expansion parameter, which is typically accompanied by the color factor $C_{A}$. The lack of convergence is in particular reflected in the huge scheme dependence of the $\mathrm{N}^{3} \mathrm{LL}$ results, i.e. the big separation of the respective MS and $\overline{\mathrm{MS}}$ curves in Fig. 2 at larger distances. Therefore we do not trust in the values for $c_{2,0}$ given above and regard them instead only as a rough order of magnitude estimate. To improve on these numbers we would need lattice data at much smaller distances. For a more detailed numerical analysis of our results see Ref. [9].

\section{Acknowledgements}

This work was partially supported by the EU network contract MRTN-CT-2006-035482 (FLAVIAnet), by the Spanish grant FPA2007-60275 and by the Catalan grant SGR2009-00894.

\section{References}

[1] W. Fischler, Nucl. Phys. B129, 157 (1977); Y. Schröder, Phys. Lett. B447, 321 (1999); B.A. Kniehl, A.A. Penin, V.A. Smirnov and M. Steinhauser, Phys. Rev. D65, 091503 (2002).

[2] C. Anzai, Y. Kiyo and Y. Sumino, arXiv:0911.4335 [hep-ph].

[3] A. V. Smirnov, V. A. Smirnov and M. Steinhauser, arXiv:0911.4742 [hep-ph].

[4] A. Pineda and J. Soto, Nucl. Phys. Proc. Suppl. 64, 428 (1998).

[5] N. Brambilla, A. Pineda, J. Soto and A. Vairo, Rev. Mod. Phys. 77, 1423 (2005).

[6] T. Appelquist, M. Dine and I. J. Muzinich, Phys. Rev. D 17, 2074 (1978).

[7] N. Brambilla, A. Pineda, J. Soto and A. Vairo, Phys. Rev. D 60, 091502 (1999).

[8] N. Brambilla, A. Pineda, J. Soto and A. Vairo, Nucl. Phys. B 566, 275 (2000).

[9] A. Pineda and M. Stahlhofen, Phys. Rev. D 81, 074026 (2010)

[10] Y. Schroder, "The static potential in QCD", DESY-THESIS1999-021.

[11] A. Pineda and J. Soto, Phys. Lett. B 420, 391 (1998).

[12] B. A. Kniehl and A. A. Penin, Nucl. Phys. B 563, 200 (1999).

[13] M. Eidemuller and M. Jamin, Phys. Lett. B 416, 415 (1998).

[14] N. Brambilla, X. Garcia i Tormo, J. Soto and A. Vairo, Phys. Lett. B 647, 185 (2007).

[15] A. Pineda and J. Soto, Phys. Lett. B 495, 323 (2000).

[16] Y. Schroder, Nucl. Phys. Proc. Suppl. 129, 572 (2004).

[17] A. Hietanen, K. Kajantie, M. Laine, K. Rummukainen and Y. Schroder, JHEP 0501, 013 (2005).

[18] A. Hietanen and A. Kurkela, JHEP 0611, 060 (2006).

[19] F. Di Renzo, M. Laine, V. Miccio, Y. Schroder and C. Torrero, JHEP 0607, 026 (2006).

[20] N. D. Hari Dass and P. Majumdar, Phys. Lett. B 658, 273 (2008).

[21] M. Luscher and P. Weisz, JHEP 0207, 049 (2002). 